\title{
И.В. Рахмелевич
}

\section{О РЕШЕНИЯХ ДВУМЕРНОГО УРАВНЕНИЯ МОНЖА - АМПЕРА СО СТЕПЕННОЙ НЕЛИНЕЙНОСТЬЮ ПО ПЕРВЫМ ПРОИЗВОДНЫМ}

\begin{abstract}
Рассматривается двумерное уравнение Монжа - Ампера, включающее нелинейность произвольного вида от искомой функции и степенные нелинейности по первым производным. Для решения данного уравнения используется метод функционального разделения переменных. Исследованы случаи, когда одна из неизвестных функций, используемых в методе разделения переменных, является линейной, а также когда эти функции являются произвольными. Проанализированы решения для различных значений параметров, характеризующих нелинейность.
\end{abstract}

Ключевые слова: уравнение Монжа - Ампера, функииональное разделение переменных, степенная нелинейность.

Уравнение Монжа - Ампера является одним из наиболее интенсивно исследуемых уравнений нелинейной математической физики благодаря многочисленным приложениям в различных областях науки, в том числе в газовой динамике, метеорологии, дифференциальной геометрии и других [1-4]. Наряду с классическим видом этого уравнения представляет интерес его дальнейшие обобщения, связанные с усложнением входящего в его состав нелинейного дифференциального оператора. Целью настоящей работы является изучение точных решений модифицированного уравнения Монжа - Ампера, правая часть которого содержит степенные нелинейности по производным и произвольную нелинейность от искомой функции. При этом в качестве основного метода исследования применяется метод разделения переменных, который остается одним из самых эффективных методов решения нелинейных дифференциальных уравнений в частных производных [5-13].

\section{1.Постановка задачи}

Рассмотрим уравнение Монжа - Ампера с нелинейной правой частью следующего вида:

$$
\frac{\partial^{2} u}{\partial x^{2}} \frac{\partial^{2} u}{\partial y^{2}}-\left(\frac{\partial^{2} u}{\partial x \partial y}\right)^{2}=g(u)\left(\frac{\partial u}{\partial x}\right)^{\beta_{1}}\left(\frac{\partial u}{\partial y}\right)^{\beta_{2}}
$$

Здесь $g(u)$ - некоторая заданная функция, $\beta_{1}, \beta_{2}$ - вещественные параметры.

Для нахождения решений уравнения (1) будем использовать функциональное разделение переменных [5] аддитивного типа, т.е. решения этого уравнения будем искать в виде

$$
\begin{gathered}
u(x, y)=U(z) \\
z=X(x)+Y(y),
\end{gathered}
$$


где $U(z), X(x), Y(y)$ - неизвестные функции, которые должны быть определены в дальнейшем. Подстановка выражений (2), (3) в уравнение (1) после некоторых преобразований дает

$$
\begin{gathered}
{\left[U^{\prime}(z)\right]^{2} X^{\prime \prime}(x) Y^{\prime \prime}(y)+U^{\prime}(z) U^{\prime \prime}(z)\left\{X^{\prime \prime}(x)\left[Y^{\prime}(y)\right]^{2}+\left[X^{\prime}(x)\right]^{2} Y^{\prime \prime}(y)\right\}=} \\
=g(U)\left[U^{\prime}(z)\right]^{\beta_{\Sigma}}\left[X^{\prime}(x)\right]^{\beta_{1}}\left[Y^{\prime}(y)\right]^{\beta_{2}} .
\end{gathered}
$$

Здесь и далее всюду $\beta_{\Sigma}=\beta_{1}+\beta_{2}$.

Рассмотрим решения уравнения (4) для частных случаев, когда одна из неизвестных функций $U(z), X(x), Y(y)$ является линейной.

1. $Y(y)=c_{2} y$. Тогда указанное уравнение принимает вид

$$
\frac{U^{\prime \prime}(z)\left[U^{\prime}(z)\right]^{1-\beta_{\Sigma}}}{g(U)}=c_{2}^{\beta_{2}-2} \frac{\left[X^{\prime}(x)\right]^{\beta_{1}}}{X^{\prime \prime}(x)}
$$

Уравнение (5) продифференцируем почленно по $y$, откуда следует уравнение для $U(z)$ :

$$
\frac{U^{\prime \prime}(z)\left[U^{\prime}(z)\right]^{1-\beta_{\Sigma}}}{g(U)}=\alpha,
$$

где $\alpha$ - некоторая постоянная. Учитывая (6), из (5) получаем уравнение для $X(x)$ :

$$
\frac{X^{\prime \prime}(x)}{\left[X^{\prime}(x)\right]^{\beta_{1}}}=A_{1}, \quad A_{1}=\frac{c_{2}^{\beta_{2}-2}}{\alpha} .
$$

Решение уравнения (7) выражается следующими формулами:

при $\beta_{1}=1$ :

$$
X(x)=X_{0}+X_{1} \exp \left(A_{1} x\right)
$$

при $\beta_{1}=2$ :

$$
X(x)=X_{0}-\frac{1}{A_{1}} \ln \left(x-x_{0}\right)
$$

при $\beta_{1} \neq 1, \beta_{1} \neq 2$ :

$$
X(x)=X_{0}+\frac{V_{1}}{\theta}\left(x-x_{0}\right)^{\theta},
$$

где $\theta=\frac{\beta_{1}-2}{\beta_{1}-1}, V_{1}=\left\{\left(1-\beta_{1}\right) A_{1}\right\} \frac{1}{1-\beta_{1}} ; X_{0}, X_{1}$ - произвольные постоянные.

Для нахождения функции $U(z)$ используем уравнение (6). Умножив это уравнение почленно на $U^{\prime}(z)$, запишем его в виде

$$
U^{\prime \prime}(z)\left[U^{\prime}(z)\right]^{2-\beta_{\Sigma}}=\alpha \frac{d}{d z} G(U),
$$

где $G(U)=\int g(U) d U$. 
Далее рассмотрим частные случаи.

Случай $a) . \beta_{\Sigma} \neq 3$.

Понизив порядок уравнения (11), приводим его к уравнению первого порядка:

$$
\left[U^{\prime}(z)\right]^{3-\beta_{\Sigma}}-\alpha\left(3-\beta_{\Sigma}\right) G(U)=A,
$$

где $A$ - произвольная постоянная.

Уравнение (12) сводится к уравнению с разделяющимися переменными, решение которого можно записать в неявной форме:

$$
z-z_{0}=\int \frac{d U}{\left[\alpha\left(3-\beta_{\Sigma}\right) G(U)+A\right]^{1 /\left(3-\beta_{\Sigma}\right)}} .
$$

Здесь и всюду далее $z_{0}$ - произвольная постоянная.

Случай б). $\beta_{\Sigma}=3$.

Тогда уравнение (11) принимает вид

$$
\frac{U^{\prime \prime}(z)}{U^{\prime}(z)}=\alpha \frac{d}{d z} G(U) .
$$

Понижая порядок уравнения (14) и решая полученное уравнение первого порядка, находим решение в неявной форме:

$$
z-z_{0}=A \int \exp (-\alpha G(U)) d U .
$$

Учитывая выражение (3), окончательно получаем следующие решения в неявной форме для случая линейной зависимости от переменной $y$ :

$$
X(x)+c_{2} y-z_{0}=\int \frac{d U}{\left[\alpha\left(3-\beta_{\Sigma}\right) G(U)+A\right]^{1 /\left(3-\beta_{\Sigma}\right)}}
$$

для $\beta_{\Sigma} \neq 3$;

$$
X(x)+c_{2} y-z_{0}=A \int \exp (-\alpha G(U)) d U
$$

для $\beta_{\Sigma}=3$.

Функция $X(x)$, входящая в (16) и (17), определяется одним из выражений (8), (9) и (10) в зависимости от значения $\beta_{1}$.

Для некоторых простейших случаев из (16) и (17) можно получить решения уравнения (1) в явном виде. Пусть $g(u)=g_{0}=\mathrm{const}$, т.е. уравнение (1) не содержит явно искомой функции. Тогда, вычисляя интегралы в (16) и (17), получим следующие решения:

1) при $\beta_{\Sigma}=2$ :

$$
u(x, y)=U_{0} \exp \left[\alpha g_{0}\left(X(x)+c_{2} y\right)\right],
$$

где $U_{0}$ - новая произвольная постоянная;

2) при $\beta_{\Sigma}=3$ :

$$
u(x, y)=-\frac{1}{\alpha g_{0}} \ln \left(X(x)+c_{2} y-z_{0}\right)
$$


3) при $\beta_{\Sigma} \neq 2, \beta_{\Sigma} \neq 3$ :

$$
u(x, y)=\frac{1}{\alpha g_{0}\left(3-\beta_{\Sigma}\right)}\left[\alpha g_{0}\left(2-\beta_{\Sigma}\right)\left(X(x)+c_{2} y-z_{0}\right)\right]^{\frac{3-\beta_{\Sigma}}{2-\beta_{\Sigma}}} .
$$

Проводя аналогичные рассуждения, можно получить решения, аналогичные приведенным выше, для случая линейной зависимости $X(x)=c_{1} x$.

2. $U(z)=z$

В этом случае уравнение (4) можно привести к виду

$$
\left\{\left[X^{\prime}(x)\right]^{-\beta_{1}} X^{\prime \prime}(x)\right\} \cdot\left\{\left[Y^{\prime}(y)\right]^{-\beta_{2}} Y^{\prime \prime}(y)\right\}=g(U) .
$$

Далее, введем обозначения

$$
\left[X^{\prime}(x)\right]^{-\beta_{1}} X^{\prime \prime}(x)=\varphi(x),\left[Y^{\prime}(y)\right]^{-\beta_{2}} Y^{\prime \prime}(y)=\psi(y),
$$

с учетом которых (18) принимает вид

$$
\varphi(x) \psi(y)=g(z)
$$

Логарифмируя (18a) и дифференцируя полученное соотношение по $x$ и по $y$, с учетом (3) получаем

$$
\frac{d^{2}}{d z^{2}} \ln g(z)=0
$$

откуда находим

$$
g(z)=g_{0} \exp (\lambda z) .
$$

Из (18) и (19) следуют уравнения для функций $X(x), Y(y)$ :

$$
\begin{aligned}
& {\left[X^{\prime}(x)\right]^{-\beta_{1}} X^{\prime \prime}(x)=A_{1} \exp (\lambda X),} \\
& {\left[Y^{\prime}(y)\right]^{-\beta_{2}} Y^{\prime \prime}(y)=A_{2} \exp (\lambda Y),}
\end{aligned}
$$

где $A_{1}, A_{2}$ - некоторые постоянные, связанные соотношением $A_{1} A_{2}=g_{0}$.

a) $\lambda=0$. Тогда первое из уравнений (20) совпадает с уравнением (7), поэтому его решение определяется формулами (8), (9) и (10). Второе из уравнений (20) также с точностью до обозначений совпадает с (7), поэтому выражения для $Y(y)$ получаются из (8), (9) и (10) путем замены $x \rightarrow y, X_{0}, X_{1} \rightarrow Y_{0}, Y_{1}, A_{1} \rightarrow A_{2}$, $\beta_{1} \rightarrow \beta_{2}$.

б) $\lambda \neq 0$. В этом случае решение первого из уравнений (20) можно записать в неявном виде

$$
x-x_{0}=\left\{\begin{array}{cc}
\int\left(\tilde{A}_{1} \exp (\lambda X)+B_{1}\right)^{1 /\left(\beta_{1}-2\right)} d X & \text { при } \beta_{1} \neq 2, \\
B_{1} \int \exp \left(\tilde{A}_{1} \exp (\lambda X)\right) d X & \text { при } \beta_{1}=2,
\end{array}\right.
$$

где $B_{1}-$ произвольная постоянная,

$$
\tilde{A}_{1}=\left\{\begin{array}{cc}
A_{1}\left(2-\beta_{1}\right) / \lambda & \text { при } \beta_{1} \neq 2, \\
A_{1} / \lambda & \text { при } \beta_{1}=2 .
\end{array}\right.
$$

Аналогичный вид имеет решение для второго уравнения (20). 


\section{2. Общий случай уравнения Монжа - Ампера со степенными нелинейностями}

В данном параграфе рассмотрим общий случай уравнения (1), причем будем предполагать, что для всех неизвестных функций выполнены условия

$$
U^{\prime}(z) \neq \text { const, } X^{\prime}(x) \neq \text { const, } Y^{\prime}(y) \neq \text { const }
$$

(альтернативные случаи рассмотрены в предыдущем разделе).

Тогда, разделив почленно уравнение (4) на $U^{\prime}(z) U^{\prime \prime}(z) X^{\prime \prime}(x) Y^{\prime \prime}(y)$, преобразуем его к виду

$$
\frac{U^{\prime}(z)}{U^{\prime \prime}(z)}+\frac{\left[X^{\prime}(x)\right]^{2}}{X^{\prime \prime}(x)}+\frac{\left[Y^{\prime}(y)\right]^{2}}{Y^{\prime \prime}(y)}=\frac{g(U)\left[U^{\prime}(z)\right]^{\beta_{\Sigma}-1}}{U^{\prime \prime}(z)} \cdot \frac{\left[X^{\prime}(x)\right]^{\beta_{1}}}{X^{\prime \prime}(x)} \cdot \frac{\left[Y^{\prime}(y)\right]^{\beta_{2}}}{Y^{\prime \prime}(y)} .
$$

Уравнение (1) допускает разделение переменных, если каждая из функций $U(z), X(x), Y(y)$ может быть определена в результате решения некоторого обыкновенного дифференциального уравнения (ОДУ). Уравнение (23) может быть сведено к ОДУ относительно $U(z)$ только в том случае, если функции $X(x), Y(y)$ удовлетворяют условиям

$$
\frac{\left[X^{\prime}(x)\right]^{2}}{X^{\prime \prime}(x)}+\frac{\left[Y^{\prime}(y)\right]^{2}}{Y^{\prime \prime}(y)}=\xi(z), \frac{\left[X^{\prime}(x)\right]^{\beta_{1}}}{X^{\prime \prime}(x)} \cdot \frac{\left[Y^{\prime}(y)\right]^{\beta_{2}}}{Y^{\prime \prime}(y)}=\eta(z),
$$

где $\xi(z), \eta(z)$ - некоторые неизвестные функции.

Дифференцируя первое из уравнений (24) по $x$ и по $y$, получаем уравнение $\xi^{\prime \prime}(z)=0$. Подставляя его решение $\xi(z)=a z+a_{0}$ в первое из уравнений (24), получаем

$$
\left(\frac{\left[X^{\prime}(x)\right]^{2}}{X^{\prime \prime}(x)}-a X(x)\right)+\left(\frac{\left[Y^{\prime}(y)\right]^{2}}{Y^{\prime \prime}(y)}-a Y(y)\right)=a_{0} .
$$

Из (25) следует, что функции $X(x), Y(y)$ должны удовлетворять уравнениям

$$
\begin{gathered}
\frac{\left[X^{\prime}(x)\right]^{2}}{X^{\prime \prime}(x)}=a X(x)+a_{1} ; \\
\frac{\left[Y^{\prime}(y)\right]^{2}}{Y^{\prime \prime}(y)}=a Y(y)+a_{2},
\end{gathered}
$$

где постоянные $a_{1}, a_{2}$ связаны соотношением $a_{1}+a_{2}=a_{0}$.

Аналогичным образом, логарифмируя второе из уравнений (24) и дифференцируя его по $x$ и по $y$, получаем

$$
\eta(z)=b \exp (\lambda z),
$$

где $b, \lambda$ - произвольные постоянные. Подставляя (27) во второе уравнение (24), с учетом (3), находим

$$
\left\{\frac{\left[X^{\prime}(x)\right]^{\beta_{1}}}{X^{\prime \prime}(x)} \cdot \exp (-\lambda X(x))\right\} \cdot\left\{\frac{\left[Y^{\prime}(y)\right]^{\beta_{2}}}{Y^{\prime \prime}(y)} \cdot \exp (-\lambda Y(y))\right\}=b .
$$


Из (28) следует вторая пара уравнений, которым должны удовлетворять $X(x), Y(y)$ :

$$
\begin{gathered}
\frac{\left[X^{\prime}(x)\right]^{\beta_{1}}}{X^{\prime \prime}(x)}=b_{1} \exp (\lambda X(x)), \\
\frac{\left[Y^{\prime}(y)\right]^{\beta_{2}}}{Y^{\prime \prime}(y)}=b_{2} \exp (\lambda Y(y)),
\end{gathered}
$$

причем постоянные $b_{1}, b_{2}$ связаны соотношением $b_{1} b_{2}=b$.

Из приведенных выше рассуждений следует, что функция $X(x)$ должна удовлетворять системе двух уравнений (26a), (29a), а функция $Y(y)$ - системе уравнений (26б), (29б), причем каждая из этих систем является переопределенной. Из (23) и (24) следует уравнение для функции $U(z)$ :

$$
\frac{U^{\prime}(z)}{U^{\prime \prime}(z)}+\xi(z)-\frac{g(U)\left[U^{\prime}(z)\right]^{\beta_{\Sigma}-1}}{U^{\prime \prime}(z)} \eta(z)=0
$$

или, с учетом выражений для $\xi(z), \eta(z)$ :

$$
U^{\prime \prime}(z)\left(a z+a_{0}\right)+U^{\prime}(z)-b g(U)\left[U^{\prime}(z)\right]^{\beta_{\Sigma}-1} \exp (\lambda z)=0 .
$$

Для нахождения функций $X(x), Y(y)$ рассмотрим системы уравнений (26а), (29a) и (26б), (29б) соответственно. Исключая $X^{\prime \prime}(x)$ из системы (26a), (29a), получаем ОДУ первого порядка:

где

$$
\begin{aligned}
& X^{\prime}(x)=\left(p_{1} X(x)+q_{1}\right)^{v_{1}} \exp \left(-\lambda_{1} X(x)\right), \\
& p_{1}=\frac{a}{b_{1}}, \quad q_{1}=\frac{a_{1}}{b_{1}}, \lambda_{1}=\frac{\lambda}{2-\beta_{1}}, v_{1}=\frac{1}{2-\beta_{1}} .
\end{aligned}
$$

Из уравнения (31) следует

$$
\frac{\left[X^{\prime}(x)\right]^{2}}{X^{\prime \prime}(x)}=\frac{p_{1} X(x)+q_{1}}{v_{1}-\lambda_{1}\left(p_{1} X(x)+q_{1}\right)} .
$$

Аналогичным образом, исключая $Y^{\prime \prime}(y)$ из системы (26б), (29б), получаем уравнение:

где

$$
\begin{aligned}
& Y^{\prime}(y)=\left(p_{2} Y(y)+q_{2}\right)^{v_{2}} \exp \left(-\lambda_{2} Y(y)\right), \\
& p_{2}=\frac{a}{b_{2}}, \quad q_{2}=\frac{a_{2}}{b_{2}}, \lambda_{2}=\frac{\lambda}{2-\beta_{2}}, v_{2}=\frac{1}{2-\beta_{2}} .
\end{aligned}
$$

Аналогично уравнению (32), из (33) находим

$$
\frac{\left[Y^{\prime}(y)\right]^{2}}{Y^{\prime \prime}(y)}=\frac{p_{2} Y(y)+q_{2}}{v_{2}-\lambda_{2}\left(p_{2} Y(y)+q_{2}\right)} .
$$

Используя уравнения (32) и (34), найдем условия совместности систем (26a), (29а) и (26б), (29б). 
Сравнивая правые части уравнений (32) и (26a), (34) и (26б), находим, что системы уравнений (26a), (29a) и (26б), (29б) являются совместными в следующих случаях:

Случай 1)

$$
\lambda=0, \frac{p_{1}}{v_{1}}=\frac{p_{2}}{v_{2}}=a, \quad \frac{q_{1}}{v_{1}}=a_{1}, \frac{q_{2}}{v_{2}}=a_{2} .
$$

Из соотношений (35) с учетом (31a) следует, что $b_{1}=2-\beta_{1}, b_{2}=2-\beta_{2}$.

Функцию $X(x)$ определяем в результате решения уравнения (31):

$$
\begin{gathered}
X(x)=k_{1} S_{1}(x)-\frac{q_{1}}{p_{1}} ; \\
S_{1}(x)=\left\{\begin{array}{cc}
\left(x-x_{0}\right)^{\frac{2-\beta_{1}}{1-\beta_{1}}} & \text { при } \beta_{1} \neq 1, \\
\exp \left(p_{1} x\right) & \text { при } \beta_{1}=1 .
\end{array}\right.
\end{gathered}
$$

При этом $k_{1}=p_{1}^{\frac{1}{1-\beta_{1}}}\left(\frac{1-\beta_{1}}{2-\beta_{1}}\right)^{\frac{2-\beta_{1}}{1-\beta_{1}}}$ в случае $\beta_{1} \neq 1$ и $k_{1}$ - произвольное в случае $\beta_{1}=1$.

Подставив (36) в уравнение (26a), находим

$$
a=2-\beta_{1} .
$$

Аналогичным образом находим функцию $Y(y)$ из уравнения (33):

$$
\begin{gathered}
Y(y)=k_{2} S_{2}(y)-\frac{q_{2}}{p_{2}} ; \\
S_{2}(y)= \begin{cases}\left(y-y_{0}\right)^{\frac{2-\beta_{2}}{1-\beta_{2}}} & \text { при } \beta_{2} \neq 1, \\
\exp \left(p_{2} y\right) & \text { при } \beta_{2}=1 .\end{cases}
\end{gathered}
$$

Здесь $k_{2}=p_{2}^{\frac{1}{1-\beta_{2}}}\left(\frac{1-\beta_{2}}{2-\beta_{2}}\right)^{\frac{2-\beta_{2}}{1-\beta_{2}}}$ в случае $\beta_{2} \neq 1$ и $k_{2}-$ произвольное в случае $\beta_{2}=1$.

Подставив (37) в уравнение (26б), находим

$$
a=2-\beta_{2} .
$$

Из сопоставления (37а) и (37б) следует, что в рассматриваемом случае решение существует, только если выполняется условие $\beta_{1}=\beta_{2}=\beta$. Отсюда с учетом (31a) и (33а) следует, что $p_{1}=p_{2}=1, k_{1}=k_{2}=k$.

Для данного случая уравнение (30) для функции $U(z)$ приводится к виду

$$
a \tilde{z} U^{\prime \prime}(\tilde{z})+U^{\prime}(\tilde{z})-b g(U)\left[U^{\prime}(\tilde{z})\right]^{\beta_{\Sigma}-1}=0 .
$$

При этом использована замена переменной $\tilde{z}=z+\frac{a_{0}}{a}$.

Рассмотрим случай, когда $g(u)=g_{0} u^{\gamma}$, т.е. уравнение содержит степенные нелинейности как по производным, так и по неизвестной функции. Покажем, что в 
этом случае уравнение (38) имеет частное решение вида

$$
U=U_{0} \tilde{z}^{\sigma} .
$$

Подставляя (39) в уравнение (38), находим

$$
\sigma=\frac{2(\beta-1)}{2 \beta+\gamma-2}, U_{0}=\frac{1}{\sigma^{\sigma}}\left(\frac{1+a(\sigma-1)}{b g_{0}}\right)^{\frac{1}{2 \beta+\gamma-2}} .
$$

Тогда из (36), (37) и (39), возвращаясь к старой переменной $z$, окончательно получаем решение уравнения (1):

$$
u(x, y)=u_{0}\left(S_{1}(x)+S_{2}(y)\right)^{\sigma},
$$

где $S_{1}(x), S_{2}(y)$ определяются выражениями (36a), (37a), $u_{0}=U_{0} k^{\sigma}$.

Рассмотрим решение при особых значениях параметров, определяемых соотношениями

$$
\begin{gathered}
\beta=1 ; \\
2 \beta+\gamma-2=0 .
\end{gathered}
$$

а) Если выполнено условие (42а), а условие (42б) не выполняется, т.е. $\beta=1$, $\gamma \neq 0$, то решение (41) вырождается в константу $u(x, y)=u_{0}$;

б) Если выполнено условие (42б), а условие (42а) не выполняется, то решение (41) не существует;

в) Если выполняются оба условия (42а), (42б), то уравнение (1) имеет решение следующего вида:

$$
u(x, y)=\left(k_{1} \exp \left(p_{1} x\right)+k_{2} \exp \left(p_{2} y\right)\right) \frac{g_{0}}{p_{1} p_{2}},
$$

где $p_{1}, p_{2}, k_{1}, k_{2}$ - произвольные постоянные.

Случай 2)

$$
a=0, \frac{q_{1}}{v_{1}-\lambda_{1} q_{1}}=a_{1}, \frac{q_{2}}{v_{2}-\lambda_{2} q_{2}}=a_{2} .
$$

Тогда из уравнений (26a) и (29a) с учетом (43) находим функцию $X(x)$; из уравнений (26б) и (29б) с учетом (43) находим функцию $Y(y)$ :

$$
\begin{aligned}
& X(x)=X_{0}-a_{1} \ln \left(x-x_{0}\right) ; \\
& Y(y)=Y_{0}-a_{2} \ln \left(y-y_{0}\right) .
\end{aligned}
$$

Здесь $x_{0}, y_{0}$ - произвольные постоянные; $X_{0}, Y_{0}$ определяются выражениями

$$
X_{0}=\frac{1}{\lambda} \ln \left(-\frac{\left(-a_{1}\right)^{\beta_{1}-1}}{b_{1}}\right), Y_{0}=\frac{1}{\lambda} \ln \left(-\frac{\left(-a_{2}\right)^{\beta_{2}-1}}{b_{2}}\right) \text {. }
$$

При этом уравнение (30) принимает вид

$$
a_{0} U^{\prime \prime}(z)+U^{\prime}(z)-b g(U)\left[U^{\prime}(z)\right]^{\beta_{1}+\beta_{2}-1} \exp (\lambda z)=0 .
$$

Таким образом, в данном случае решение (2) содержит функции $X(x), Y(y)$, определяемые выражениями (44), (45) и функцию $U(z)$, являющуюся решением уравнения (46). 
Случай 3)

$$
\beta_{1}=\beta_{2}=2, \lambda=0, \quad a=0, \quad b_{1}=a_{1}, b_{2}=a_{2} .
$$

Тогда уравнение (30) упрощается так:

$$
a_{0} U^{\prime \prime}(z)+U^{\prime}(z)-b g(U)\left[U^{\prime}(z)\right]^{3}=0,
$$

а функции $X(x), Y(y)$ находим из уравнений (26а) и (26б) с учетом (47). Решая уравнение (48) и опуская промежуточные преобразования, находим

$$
\begin{gathered}
u(x, y)= \pm a_{0}\left(\frac{1}{b g_{0}}\right)^{1 / 2} \arcsin (w(x, y)) \text { при } a_{0} \neq 0, b g_{0}>0 ; \\
u(x, y)= \pm a_{0}\left(-\frac{1}{b g_{0}}\right)^{1 / 2} \ln \left\{w(x, y)+\sqrt{1+w^{2}(x, y)}\right\} \text { при } a_{0} \neq 0, b g_{0}<0 ; \\
u(x, y)= \pm a_{0}\left(\frac{1}{b g_{0}}\right)^{1 / 2}\left\{a_{1} \ln \left|x-x_{0}\right|+a_{2} \ln \mid y-y_{0}\right\} \text { при } a_{0}=0, b g_{0}<0,
\end{gathered}
$$

где $w(x, y)=C_{0}\left(x-x_{0}\right)^{-a_{1} / a_{0}}\left(y-y_{0}\right)^{-a_{2} / a_{0}}$. Решения уравнения (1), определяемые формулами(49) - (51), получены в предположении, что $g(u)=g_{0}$.

\section{Заключение}

Таким образом, в данной работе исследовано двумерное уравнение Монжа Ампера, содержащее степенные нелинейности по первым производным и нелинейность произвольного вида по неизвестной функции. С помощью метода функционального разделения переменных получен ряд точных решений данного уравнения. В частности, рассмотрен случай, когда одна из функций $U(z), X(x), Y(y)$, вводимых при разделении переменных, является линейной, а также общий случай, когда указанные функции могут быть произвольными. Получены точные решения в явном виде для уравнения, не содержащего явно искомой функции. Проанализирован вид решений в зависимости от значений параметров, характеризующих нелинейность.

\section{ЛИТЕРАТУРА}

1. Полянин А.Д., Зайщев В.Ф. Справочник по нелинейным уравнениям математической физики: точные решения. М.: Физматлит, 2002. 432 с.

2. Хабиров C.В. Неизэнтропические одномерные движения газа, построенные с помощью контактной группы уравнения Монжа - Ампера // Математический сборник. 1990. T. 181. № 12. С. 1607-1622.

3. Шабловский О.Н. Параметрические решения уравнения Монжа - Ампера и течения газа с переменной энтропией // Вестник Томского государственного университета. Математика и механика. 2015. № 1(33). С. 105-118. DOI 10.17223/19988621/33/11.

4. Кушнер А.Г. Контактная линеаризация уравнений Монжа - Ампера и инварианты Лапласа // Доклады РАН. 2008. Т. 422. № 5. С. 1-4.

5. Полянин А.Д., Зайщев В.Ф., Журов А.И. Методы решения нелинейных уравнений математической физики и механики. М.: Физматлит, 2005. $256 \mathrm{c}$.

6. Полянин А.Д., Журов А.И. Обобщенное и функциональное разделение переменных в математической физике и механике// Доклады РАН. 2002. Т. 382. № 5. С. 606-611. 
7. Рахмелевич И.В. О применении метода разделения переменных к уравнениям математической физики, содержащим однородные функции от производных // Вестник Томского государственного университета. Математика и механика. 2013. № 3(23). С. 37-44.

8. Рахмелевич И.В. Об уравнениях математической физики, содержащих мультиоднородные функции от производных // Вестник Томского государственного университета. Математика и механика. 2014. № 1. С. 42-50.

9. Рахмелевич И.В. О решениях многомерного уравнения Клеро с мультиоднородной функцией от производных // Известия Саратовского университета. Новая серия. Серия Математика, механика, информатика. 2014. Т. 14. № 4-1. С. 374-381.

10. Рахмелевич И.В. О двумерных гиперболических уравнениях со степенной нелинейностью по производным // Вестник Томского государственного университета. Математика и механика. 2015. № 1(33). С. 12-19. DOI 10.17223/19988621/33/2.

11. Рахмелевич И.В. О некоторых новых решениях многомерного уравнения в частных производных первого порядка со степенными нелинейностями // Вестник Томского государственного университета. Математика и механика. 2015. № 3(35). С. 18-25. DOI $10.17223 / 19988621 / 35 / 3$.

12. Miller J. (Jr.), Rubel L.A. Functional separation of variables for Laplace equations in two dimensions // Journal of Physics A. 1993. V. 26. P. 1901-1913.

13. Zhdanov R.Z. Separation of variables in the non-linear wave equation // Journal of Physics A. 1994. V. 27. P. L291-L297.

Статья поступила 16.03.2016 г.

Rakhmelevich I.V. (2016) ON SOLUTIONS OF THE MONGE - AMPERE EQUATION WITH POWER-LAW NON-LINEARITY WITH RESPECT TO FIRST DERIVATIVES. Tomsk State University Journal of Mathematics and Mechanics. 4(42). pp. 33-43

\section{DOI $10.17223 / 19988621 / 42 / 4$}

In this work, the two-dimensional Monge - Ampere equation the right-hand side of which includes arbitrary non-linearity with respect to the unknown function and power-law nonlinearity with respect to its first derivatives is considered. To solve this equation, the method of functional separation of variables is used. We study the case when one of the unknown functions used in the method of separation of variables is linear and also the case when all these functions are arbitrary. The exact solutions in the implicit form of the considered equation are received in the presence of arbitrary nonlinearity with respect to the unknown function. For the case when the equation does not contain unknown function explicitly, its solutions are found in an explicit form. The solutions have been analyzed for different values of parameters characterizing the nonlinearity.

Keywords: Monge - Ampere equation, functional separation of variables, power-law non-linearity.

RAKHMELEVICH Igor Vladimirovich (Candidate of Technical Sciences, Assoc. Prof., Nizhny Novgorod State University, Nizhny Novgorod, Russian Federation)

E-mail: igor-kitpd@yandex.ru

\section{REFERENCES}

1. Polyanin A.D. and Zaitsev V.F. (2004) Handbook of Nonlinear Partial Differential Equations. Chapman \& Hall/CRC Press, Boca Raton.

2. Khabirov S.V. (1992) Nonisentropic one-dimensional gas motions constructed by means of the contact group of the nonhomogeneous Monge - Ampère equation. Mathematics of the USSR - Sbornik. 71(2). pp. 447-462. DOI 10.1070/SM1992v071n02ABEH001405.

3. Shablovsky O.N. (2015) Parametricheskie resheniya uravneniya Monzha-Ampera i techeniya gaza s peremennoy entropiey [Parametric solutions for the Monge - Ampere equation and gas flows with variable entropy]. Vestnik Tomskogo gosudarstvennogo universiteta. Matematika $i$ mekhanika - Tomsk State University Journal of Mathematics and Mechanics. 1(33). pp. 105-118. DOI 10.17223/19988621/33/11. 
4. Kushner A.G. (2008) Transformation of hyperbolic Monge - Ampére equations into linear equations with constant coefficients. Doklady Mathematics.78(3). pp. 907-909.

5. Polyanin A.D., Zaitsev V.F., Zhurov A.I. (2005) Metody resheniya nelineinyh uravneniy matematicheskoy fiziki i mekhaniki [Methods of solving nonlinear equations of mathematical physics and mechanics]. Moscow: Fizmatlit Publ.

6. Polyanin A.D., Zhurov A.I. (2002) The generalized and functional separation of variables in mathematical physics and mechanics. Doklady Mathematics. 65(1). pp. 129-134.

7. Rakhmelevich I.V. (2013) O primenenii metoda razdeleniya peremennykh k uravneniyam matematicheskoy fiziki, soderzhashchim odnorodnye funktsii ot proizvodnykh [On application of the variable separation method to mathematical physics equations containing homogeneous functions of derivatives]. Vestnik Tomskogo gosudarstvennogo universiteta. Matematika i mekhanika - Tomsk State University Journal of Mathematics and Mechanics. 3(23). pp. 37-44.

8. Rakhmelevich I.V. (2014) Ob uravneniyakh matematicheskoy fiziki, soderzhashchikh mul'tiodnorodnye funktsii ot proizvodnykh [On equations of mathematical physics containing multi-homogeneous functions of derivatives]. Vestnik Tomskogo gosudarstvennogo universiteta. Matematika i mekhanika - Tomsk State University Journal of Mathematics and Mechanics. 1(27). pp. 42-50.

9. Rakhmelevich I.V. (2014) O resheniyakh mnogomernogo uravneniya Klero s mul'tiodnorodnoy funktsiey ot proizvodnykh [On the Solutions of Multi-dimensional Clairaut Equation with Multi-homogeneous Function of the Derivatives]. Izvestiya Saratovskogo universiteta. Novaya seriya. Seriya Matematika, mekhanika, informatika. 14(4-1). pp. 374-381.

10. Rakhmelevich I.V. (2015) O dvumernykh giperbolicheskikh uravneniyakh so stepennoy nelineynost'yu po proizvodnym [On two-dimensional hyperbolic equations with power-law nonlinearity in the derivatives]. Vestnik Tomskogo gosudarstvennogo universiteta. Matematika i mekhanika - Tomsk State University Journal of Mathematics and Mechanics.1(33). pp. 12-19. DOI 10.17223/19988621/33/2.

11. Rakhmelevich I.V. (2015) O nekotorykh novykh resheniyakh mnogomernogo uravneniya $\mathrm{v}$ chastnykh proizvodnykh pervogo poryadka so stepennymi nelineynost'yami [On some new solutions of the multi-dimensional first order partial differential equation with power-law nonlinearities]. Vestnik Tomskogo gosudarstvennogo universiteta. Matematika i mekhanikaTomsk State University Journal of Mathematics and Mechanics. 3(35). pp. 18-25. DOI $10.17223 / 19988621 / 35 / 3$.

12. Miller J. (Jr.), Rubel L.A. (1993) Functional separation of variables for Laplace equations in two dimensions. Journal of Physics A. 26. pp. 1901-1913.

13. Zhdanov R.Z. (1994) Separation of variables in the non-linear wave equation. Journal of Physics A. 27. pp. L291-L297. 